\title{
Selbsteinschätzung körperlicher Aktivität: Der Vergleich von subjektiver und objektiver körperlicher Aktivität bei Rückenpatienten nach stationärer Rehabilitation
}

\author{
Assessing Physical Activity in Low Back Pain Patients After Inpatient Rehabilitation: \\ A Comparison of a Direct Versus a Self-report Measure
}

Autoren

Institute

\section{A. Schaller, K. Rudolf, F. Arndt, I. Froboese}

Deutsche Sporthochschule Köln, Institut für Bewegungstherapie und bewegungsorientierte Prävention und Rehabilitation, Köln

Schlüsselwörter
Selbsteinschätzung
subjektive körperliche
Aktivität
Akzelerometer
Rückenschmerzpatiente
Key words
self-assessment
self-reported physical
activity
accelerometer
low back pain

eingereicht: $\quad 11.08 .2015$ angenommen: 04.12.2015

Bibliografie

DOI http://dx.doi.org/ 10.1055/s-0041-111035 Phys Med Rehab Kuror 2016; 26: 71-78

(c) Georg Thieme Verlag KG Stuttgart · New York ISSN 0940-6689

Korrespondenzadresse Dr. A. Schaller German Sport University Institute of health promotion and clinical movement science Am Sportpark Muengersdorf 6 50933 Cologne

a.schaller@dshs-koeln.de

\section{Zusammenfassung \\ $\nabla$}

Fragestellung: Fragestellung der vorliegenden Studie ist der Zusammenhang sowie der Grad der Übereinstimmung zwischen subjektiver und objektiver körperlicher Aktivität bei postrehabilitativen Rückenpatienten.

Material und Methoden: 27 TeilnehmerInnen trugen über 7 Tage einen Akzelerometer (Actigraph GT3X+) und füllten einen Fragebogen (GPAQ) zur körperlichen Aktivität im gleichen Zeitraum aus. Zusammenhänge im Bereich der moderaten Aktivität, intensiven Aktivität und der Sitzzeiten wurden jeweils mittels Korrelationskoeffizienten und der Grad der Übereinstimmung mittels Bland-Altman-Analyse geprüft.

Ergebnisse: Es zeigten sich keine signifikanten Zusammenhänge. Die subjektiven Angaben zeigten im Vergleich zur objektiven Aktivität Überschätzungen von $46 \mathrm{~min} / \mathrm{Tag}$ (intensive Aktivität) bis $78 \mathrm{~min} /$ Tag (moderate Aktivität). Sitzzeiten wurden um 249 min/Tag unterschätzt.

Diskussion: Die vorliegenden Ergebnisse zum geringen Zusammenhang von subjektiver und objektiver körperlicher Aktivität bei Rückenpatienten entsprechen vergleichbaren Studien mit anderen Studienpopulationen.

Schlussfolgerungen: Aufgrund der Bedeutung der aktivitätsbezogenen Selbsteinschätzung für die Interventionskonzeption und -evaluation sollte diesem Thema in der Bewegungsförderung mehr Bedeutung beigemessen werden.

\section{Einleitung}

$\nabla$

Die Hinführung zu einem körperlich aktiven Lebensstil ist ein übergeordnetes Ziel der Bewegungstherapie und rehabilitativen Nachsorge $[1,2]$, das auch bei der großen Gruppe von Rehabilitanden mit chronischen Rückenschmerzen von zentraler Bedeutung ist [3-5].

\section{Abstract \\ $\nabla$}

Purpose: Aim of the present study was to determine the closeness of agreement between self-reported physical activity and an objective measure of physical activity in patients with low back pain.

Materials and Methods: 27 subjects with LBP wore an accelerometer (Actigraph GT3X+) for 7 consecutive days and answered a questionnaire on physical activity (GPAQ) over the same period of time. Spearman's correlation and Bland-Altman analysis for the agreement between measures for moderate and vigorous intensity as well as for sedentary behavior were calculated.

Results: There were weak no -significant correlations between subjective and objective results. Self-reported data showed an overestimation of $46 \mathrm{~min} /$ day (vigorous intensity) to $78 \mathrm{~min} /$ day (moderate intensity). Sedentary time was underestimated $249 \mathrm{~min} /$ day.

Discussion: The present results go in line with similar studies that focus different populations.

Conclusions: As the self-reported physical activity can be a decisive factor in altering the level of physical activity it should be focused in the development and evaluation of measures promoting physical activity. 
stimmung von subjektiver und objektiver körperlicher Aktivität, die Motivation und Intention zur Veränderung des Bewegungsverhaltens maßgeblich beeinflussen kann $[6,7]$. So kann eine Überschätzung des eigenen Aktivitätsverhaltens eine potentielle Barriere für den Erfolg von Interventionen sein, da sich TeilnehmerInnen unter Umständen bereits als ausreichend aktiv einschätzen und somit eine bewegungsbezogene Verhaltensveränderung nicht als notwendig empfinden [6-9].

Aus wissenschaftlicher Sicht ist für die Bewertung von Interventionseffekten neben den Fragen nach der Objektivität, Reliabilität und Validität eines Instruments zur Messung körperlicher Aktivität auch die Frage nach dessen Anwendbarkeit in der Praxis von zentraler Bedeutung [10]. Bezüglich der Operationalisierung von körperlicher Aktivität wird dabei in der Literatur ein inverser Zusammenhang zwischen Messgenauigkeit und Praktikabilität beschrieben, d.h. mit steigender Praktikabilität nimmt die Messpräzision ab [11]. ๑ Abb. 1 zeigt Methoden zur Operationalisierung körperlicher Aktivität und unterscheidet dabei Referenz-Methoden, objektive Verfahren und subjektive Verfahren. Während die Messpräzision von den subjektiven Verfahren in Richtung Referenz-Methoden zunimmt, nimmt die Praktikabilität, d.h. die Durchführung in der Routineanwendung, gleichzeitig ab $[10,12]$. Die Herausforderung in der anwendungsorientierten Wissenschaft besteht darin, für die jeweilige Untersuchung ein praktikables Instrument mit bestmöglicher Messpräzision auszuwählen.

Ausgehend von der Einteilung in $\bullet$ Abb. 1 zeigten bereits mehrere Studien, dass Daten zur körperlichen Aktivität aus subjektiven und objektiven Verfahren häufig nicht übereinstimmen. Prince et al. [13] zeigten dazu in einem umfassenden Review, dass es bei den subjektiven Erhebungen sowohl zu deutlichen Über- als auch Unterschätzungen im Vergleich zu objektiven Daten kam. Dabei konnte allerdings kein klares Muster bezüglich der Abweichungen identifiziert werden [13]. Auch Gaede-Illig, Alfermann, Zachariae und Menzel [14] zeigten in einer Studie an über 430 Probanden, dass subjektive Angaben stark von objektiven Werten zur körperlichen Aktivität abwichen, wobei sie im Mittel eine subjektive Überschätzung der körperlichen Aktivität beschreiben [14]. Zu den gleichen Ergebnissen kam ein Review zur Validität eines international anerkannten Fragebogens (IPAQSF), das eine zum Teil deutliche Überschätzung der subjektiven körperlichen Aktivität im Vergleich zu objektiven Daten beschreibt [15]. Analog zur Tendenz der Überschätzung der subjektiven körperlichen Aktivität zeigt die Studienlage zudem eine

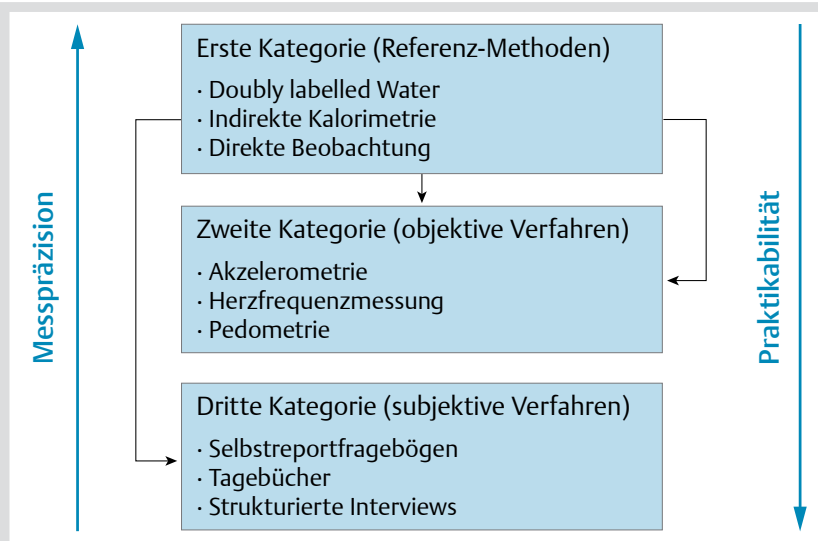

Abb. 1 Messverfahren körperlicher Aktivität (modifiziert nach Beneke \& Leithäuser, 2008 und Müller, 2010 [10,12]) zum Teil deutliche Unterschätzung von Sitzzeiten bzw. körperlicher Inaktivität [16-18]. Die Notwendigkeit einer ergänzenden indikationsspezifischen Betrachtung des Themas zeigt eine Arbeit von van Weering, Vollenbroek-Hutten und Hermens [19], die zeigte, dass die Selbsteinschätzung von Rückenschmerzpatienten im Vergleich zu gesunden Probanden deutlich schlechter ist [19]. Ausgehend von der in der Literatur aufgezeigten Problemlage bei der Übereinstimmung subjektiver und objektiver Verfahren sowie der Bedeutung der patientenbezogenen Selbsteinschätzung für die Interventionskonzeption und -evaluation von Bewegungsförderungsmaßnahmen ist das Ziel der vorliegenden Studie die subjektiven und objektiven körperlichen Aktivität bei Patienten mit Rückenschmerzen zu vergleichen.

\section{Material und Methodik \\ $\nabla$}

\section{Studiendesign}

Die Studie wurde als Teilprojekt im Rahmen eines Forschungsprojekts zur Förderung körperlicher Aktivität und Teilhabe durchgeführt [20]. Erhebungszeitraum war Juni-Juli 2014 und das Forschungsprojekt wurde durch die Ethik-Kommission der Deutschen Sporthochschule Köln genehmigt.

In einer Informationsveranstaltung erhielten die TeilnehmerInnen für 7 Tage einen Akzelerometer zur objektiven Erfassung körperlicher Aktivität. Am Ende der Tragedauer beantworteten die TeilnehmerInnen einen Fragebogen zur körperlichen Aktivität in den letzten 7 Tagen. Inhalte der Informationsveranstaltung waren die Aufklärung über die Messinstrumente und deren Handhabung, die Messung des Körpergewichts und das Unterschreiben der Einwilligungserklärung.

\section{Stichprobe}

Die Stichprobe bestand aus Rehabilitanden, die vor mindestens 6 Monaten eine stationäre Rehabilitation wegen Rückenschmerzen durchgeführt hatten. Ausschlusskriterien für das gesamte Forschungsprojekt waren unzureichende Deutschkenntnisse, posttraumatische Zustände (z. B. Rückenbeschwerden als Unfallfolge), operative Eingriffe in den letzten 3 Monaten vor der Rehabilitation, Alter über 65 Jahre und eine geplante Operation im Anschluss an die stationäre Rehabilitation [20]. Einschlusskriterium für die vorliegende Querschnittstudie war eine mindestens seit 6 Monaten abgeschlossene stationäre orthopädische Rehabilitation, keine operativen Eingriffe im Anschluss an die Rehabilitation, die Bereitschaft, einen Bewegungssensor zu tragen und eine unterschriebene Einverständniserklärung. Zudem wurden nur TeilnehmerInnen in die Auswertung eingeschlossen, die den Bewegungssensor mindestens 10 Stunden an 4 verschiedenen Tagen getragen hatten $[21,22]$.

\section{Messinstrumente}

Die Operationalisierung der subjektiven körperlichen Aktivität erfolgte mittels Global Physical Activity Questionnaire (GPAQ) $[23,24]$. Der GPAQ wird von der WHO zur Erfassung der körperlichen Aktivität empfohlen [23-26] und besteht aus 16 Items. Umfang und Intensität (moderat bzw. intensiv) von körperlicher Aktivität werden in den 3 Lebensbereichen Arbeit, Transport und Freizeit erhoben. Zudem wird in einem Item die tägliche Sitzzeit erfasst.

Die Quantifizierung der objektiven körperlichen Aktivität erfolgte anhand eines triaxialen Akzelerometers (Actigraph GT3X+). Dieser wurde während der Wachzeiten auf Höhe der Spina iliaca 
anterior getragen. Der Actigraph GT3X+misst leichte, moderate und intensive körperliche Aktivität sowie sitzende Aktivität.

Alter, Geschlecht und Schulabschluss der TeilnehmerInnen wurden mittels Fragebogen erhoben. Zudem wurde nach ungewöhnlichen Ereignissen im Erhebungszeitraum gefragt („Ist die letzte Woche eine gewöhnliche Woche gewesen?“ (ja/nein), „Sind Sie Ihren üblichen Tätigkeiten nachgegangen?“ (ja/nein)).

\section{Statistische Auswertung}

Im Rahmen der Datenaufbereitung wurden die Akzelerometerdaten anhand der Freedson- und Troiano-Algorithmen [27,28] ausgelesen und validiert. Sowohl für subjektive (GPAQ) ${ }^{1}$ als auch für objektive Daten (Akzelerometer) ${ }^{2}$ wurden Tagesdurchschnittswerte für moderate und intensive Aktivität sowie die Sitzzeit berechnet, um Probanden mit unterschiedlichen Tragezeiten vergleichbar zu machen.

Die Stichprobenbeschreibung und deskriptive Ergebnisdarstellung zur körperlichen Aktivität erfolgte auf Grundlage der Gesamtstichprobe anhand von Mittelwert (MW), Standardabweichung (SD) und Median (MED).

Die statistische Überprüfung des Zusammenhangs der subjektiven (GPAQ) und objektiven (Akzelerometer) Aktivitätsdaten erfolgte anhand des Rang-Korrelationskoeffizienten nach Spearman (rho). Dabei wurden Korrelationen bis 0,3 als geringer, über 0,3-0,7 als mittelstark und über 0,7 als starker Zusammenhang bewertet. Es wurden jeweils die subjektiven und objektiven Daten der moderaten Aktivität, der intensiven Aktivität sowie Sitzzeiten verglichen. Ergänzend wurde ein Streudiagramm erstellt, wobei TeilnehmerInnen mit Abweichungen außerhalb eines Intervalls von $\pm 10 \%$ des jeweiligen objektiven Mittelwerts als Über- bzw. Unterschätzer klassifiziert wurden. Aufgrund fehlender Standards bei wurde das Toleranzintervall wurde aus praxisorientierter Perspektive auf Grundlage einer Diskussion von Sportwissenschaftlern und Therapeuten festgelegt.

Um neben Zusammenhängen auch den Grad an Übereinstimmung zwischen subjektiver und objektiver Aktivität zu identifizieren, wurde zudem eine Bland-Altman-Analyse durchgeführt, wobei die durchschnittliche Differenz (MW) sowie die Übereinstimmungsgrenzen (1,96-faches der Standardabweichung) dargestellt wurden $[29,30]$.

Die Datenauswertung erfolgte für die Gesamtstichprobe und getrennt nach Geschlechtern. Unterschiede zwischen den Geschlechtern wurden mittels Mann-Whitney-U-Test überprüft. TeilnehmerInnen, die eine Tragedauer des Akzelerometers von mindestens 10 Stunden an 4 Tagen unterschritten hatten, wurden bei der Evaluation nicht berücksichtigt.

Signifikanztests wurden mit einem Signifikanzniveau von $p \leq$ 0,05 berechnet. Die Auswertung wurde mit IBM SPSS Statistics $22 \circledast$ durchgeführt.

\section{Ergebnisse}

\section{Stichprobenbeschreibung}

Die Auswahlpopulation bestand aus 55 potentiellen TeilnehmerInnen. Davon konnten 27 TeilnehmerInnen (49\%) in die Studie eingeschlossen werden. Bei 25 ProbandInnen konnten subjektive und objektive Datenpaare ausgewertet werden ( $\bullet$ Abb. 2).

\footnotetext{
${ }^{1}$ Wöchentliche Aktivität $/ 7=$ durchschnittliche Tagesaktivität

${ }^{2}$ Wöchentliche Aktivität/Tragedauer = durchschnittliche Tagesaktivität
}

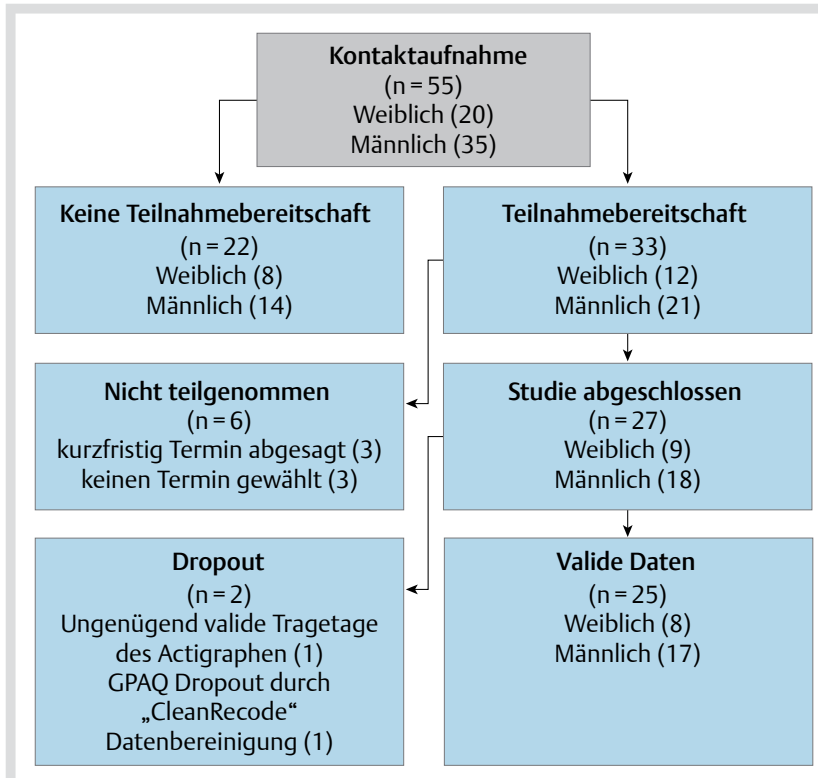

Abb. 2 Flussdiagramm: TeilnehmerInnen-Rekrutierung und Auswertung

Tab. 1 Stichprobenbeschreibung.

\begin{tabular}{|c|c|c|c|}
\hline & $\begin{array}{l}\text { Männer } \\
(n=18)\end{array}$ & $\begin{array}{l}\text { Frauen } \\
(n=9)\end{array}$ & $\mathbf{p}$ \\
\hline Alter (Jahre) [MW (SD)] & $52( \pm 7)$ & $53( \pm 7)$ & $0,86^{1}$ \\
\hline BMI [MW(SD)] & $31( \pm 5)$ & $27( \pm 3)$ & $0,23^{1}$ \\
\hline Höchster Schulabschluss „Hauptschule“ (n;\%) & $14(82 \%)$ & $4(44 \%)$ & $0,05^{2 *}$ \\
\hline $\begin{array}{l}\text { „Ist die letzte Woche eine gewöhnliche } \\
\text { Woche gewesen?“ (ja) (n;\%) }\end{array}$ & $13(72 \%)$ & $7(78 \%)$ & $0,76^{2}$ \\
\hline $\begin{array}{l}\text { „Sind Sie Ihren üblichen Tätigkeiten } \\
\text { nachgegangen?“ }\end{array}$ & $15(83 \%)$ & $7(78 \%)$ & $0,73^{2}$ \\
\hline
\end{tabular}

- Tab. 1 gibt einen Überblick über die Stichprobe aus 18 Männern und 9 Frauen. Geschlechtsspezifische Unterschiede zeigten sich beim Bildungsabschluss, wo Männer signifikant häufiger Hauptschulabschluss als höchsten Schulabschluss genannt haben $(p=0,05)$. Bezüglich des Untersuchungszeitraums gaben 20 von 27 TeilnehmerInnen an, dass es sich um eine gewöhnliche Woche in ihrem Alltag handelte und 22 von 27 TeilnehmerInnen, dass sie im Erhebungszeitraum ihren üblichen Tätigkeiten nachgegangen seien.

\section{Subjektive und objektive körperliche Aktivität}

- Tab. 2 fasst die deskriptiven Ergebnisse zur subjektiven und objektiven körperlichen Aktivität zusammen. Bei den subjektiven Angaben zur körperlichen Aktivität zeigten sich keine signifikanten geschlechtsbezogenen Unterschiede.

Die objektiven Daten zur körperlichen Aktivität zeigten bezogen auf die Sitzzeit einen signifikanten Geschlechtsunterschied $(p=0,04)$, mit längeren Sitzzeiten bei den Männern (565 vs. $477 \mathrm{~min} / \mathrm{Tag})$.

\section{Zusammenhang zwischen subjektiver und objektiver körperlicher Aktivität}

- Tab. 3 zeigt die Korrelationen subjektiver und objektiver körperliche Aktivität bei moderater bzw. intensiver Aktivität sowie den Sitzzeiten. Dabei zeigte sich in keinem Bereich in der Gesamtstichprobe oder im Rahmen der geschlechtsspezifischen 
Tab. 2 Subjektive und objektive körperliche Aktivität.

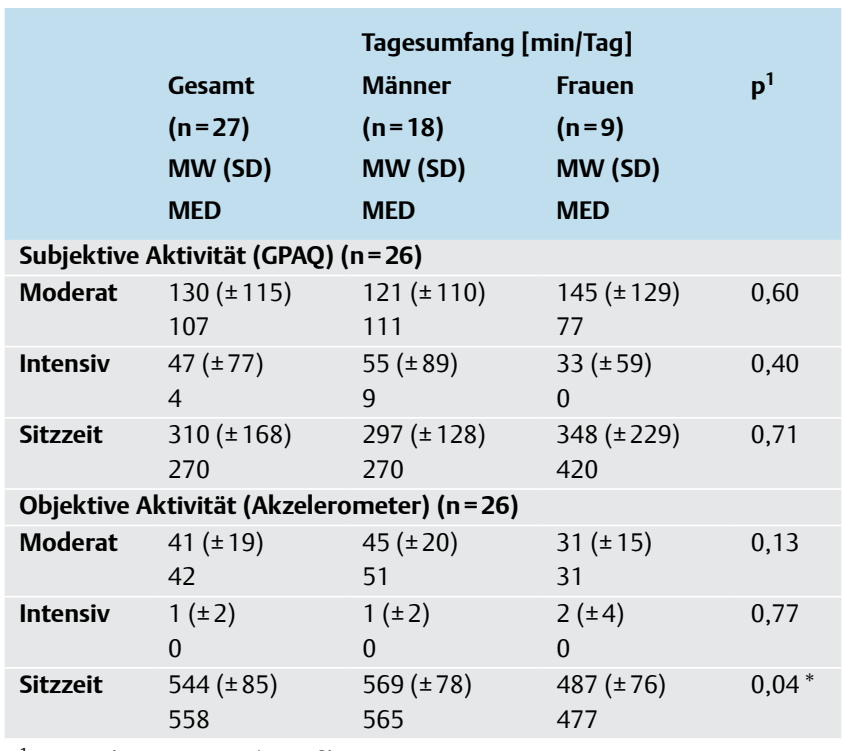

${ }^{1}$ Mann-Whitney-U-Test; * Signifikanzniveau $\mathrm{p} \leq 0,05$

Tab. 3 Korrelationskoeffizienten (rho) zur Übereinstimmung subjektiver und objektiver körperlicher Aktivität

\begin{tabular}{|c|c|c|c|}
\hline & $\begin{array}{l}\text { Gesamtstichprobe } \\
(n=25)\end{array}$ & $\begin{array}{l}\text { Männer } \\
(\mathrm{n}=17)\end{array}$ & $\begin{array}{l}\text { Frauen } \\
(n=8)\end{array}$ \\
\hline Moderate Aktivität [rho $(p)^{1}$ ] & $\begin{array}{l}-0,01 \\
(0,98)\end{array}$ & $\begin{array}{l}0,06 \\
(0,81)\end{array}$ & $\begin{array}{l}-0,55 \\
(0,16)\end{array}$ \\
\hline Intensive Aktivität [rho (p) ${ }^{1}$ ] & $\begin{array}{l}-0,17 \\
(0,42)\end{array}$ & $\begin{array}{l}0,02 \\
(0,93)\end{array}$ & $\begin{array}{l}-0,53 \\
(0,18)\end{array}$ \\
\hline Sitzzeit [rho $(p)^{1}$ ] & $\begin{array}{c}-0,04 \\
(0,86)\end{array}$ & $\begin{array}{c}-0,22 \\
(0,39)\end{array}$ & $\begin{array}{l}0,30 \\
(0,47)\end{array}$ \\
\hline
\end{tabular}

${ }^{1}$ Rang-Korrelationskoeffizienten nach Spearman; ${ }^{*}$ Signifikanzniveau $\mathrm{p} \leq 0,05$

Auswertung eine signifikante Korrelation zwischen subjektiver und objektiver Aktivität. Auffällig waren die negativen Korrelationen bei Frauen im Vergleich zu Männern, welche positive Korrelationen aufweisen. Für die Sitzzeiten ist dieses Phänomen umgekehrt, Männer zeigen einen negativen, Frauen einen positiven schwachen Zusammenhang. Während die Zusammenhänge bei Männern durchweg als gering zu bezeichnen sind (rho $=-0,22-$ 0,06 ), zeigten sich bei Frauen geringe bis mittlere Zusammenhänge zwischen subjektiven und objektiven Werten (rho=-0,55-0,30).

\section{Abweichungen zwischen subjektiver und objektiver Aktivität}

Wie $\odot$ Tab. 3 zeigt, lag zwischen der subjektiven und der objektiven moderaten körperlichen Aktivität im Tagesmittel eine sehr schwache negative Korrelation vor (rho=-0,01). Das Streudiagramm zu den Ergebnissen der moderaten körperlichen Aktivität im Tagesmittel ( $\bullet$ Abb. 3) zeigt, dass, ausgehend vom Referenzintervall von $\pm 10 \%$ des objektiven Mittelwerts, 24 TeilnehmerInnen (96\%) außerhalb des Intervalls lagen. 16 TeilnehmerInnen (64\%) lagen oberhalb des Intervalls und sind damit als „Überschätzer“ zu klassifizieren und 8 Teilnehmer (32\%) als Unterschätzer. Frauen (75\%) überschätzten ihre moderate körperliche Aktivität dabei prozentual häufiger als Männer (59\%).

- Abb. 4 stellt die Bland-Altman Analyse zur individuellen Abweichung für die moderate körperliche Aktivität im Tagesmittel dar. Die mittlere Differenz zwischen den objektiven und subjektiven Daten (Actigraph - GPAQ) betrug-79 min (SD=110), d.h.

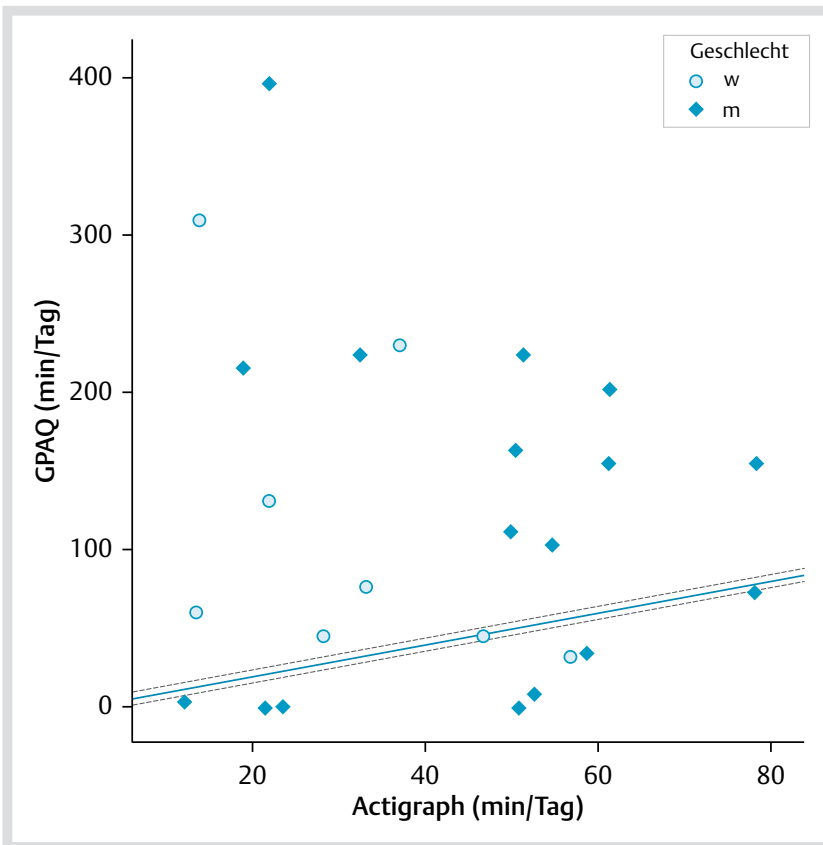

Abb. 3 Zusammenhang zwischen objektiver (Actigraph) und subjektiver (GPAQ) moderater körperlicher Aktivität im Tagesmittel. Referenzintervall: $\pm 4,1 \mathrm{~min}(10 \%)$

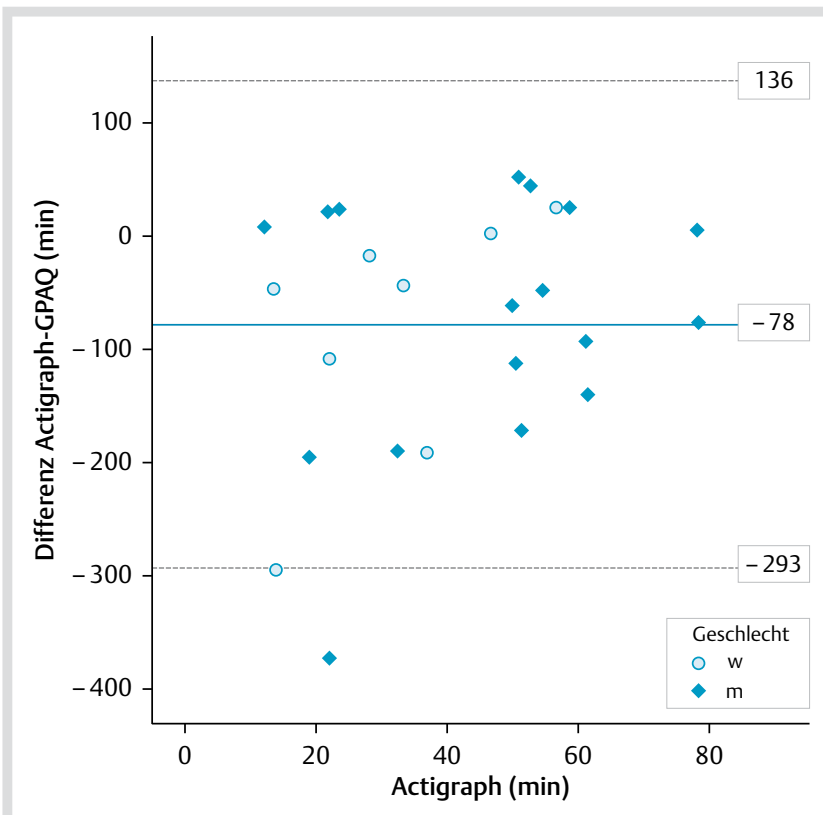

Abb. 4 Bland-Altman-Plot zur moderaten körperlichen Aktivität

im Mittel überschätzen die TeilnehmerInnen die Dauer ihrer moderaten körperlichen Aktivität um mehr als eine Stunde pro Tag. Die Abweichungen unterscheiden sich nicht signifikant zwischen männlichen und weiblichen Probanden $(p=0,84)$.

Zwischen der subjektiven und objektiven intensiven körperlichen Aktivität zeigte sich im Tagesmittel $(r=-0,17)$ eine sehr schwache negative Korrelation in der Gesamtstichprobe ( 0 Tab. 3). Das Streudiagramm in $\odot$ Abb. 5 illustriert die Zusammenhänge der intensiven körperlichen Aktivität im Tagesmittel. Zur Kategorisierung der Einschätzung wurde ein Intervall von $\pm 10 \%$ des objektiven Mittelwerts gesetzt. Insgesamt lagen 18 TeilnehmerInnen (72\%) außerhalb des Intervalls, wobei sich 6 TeilnehmerIn- 


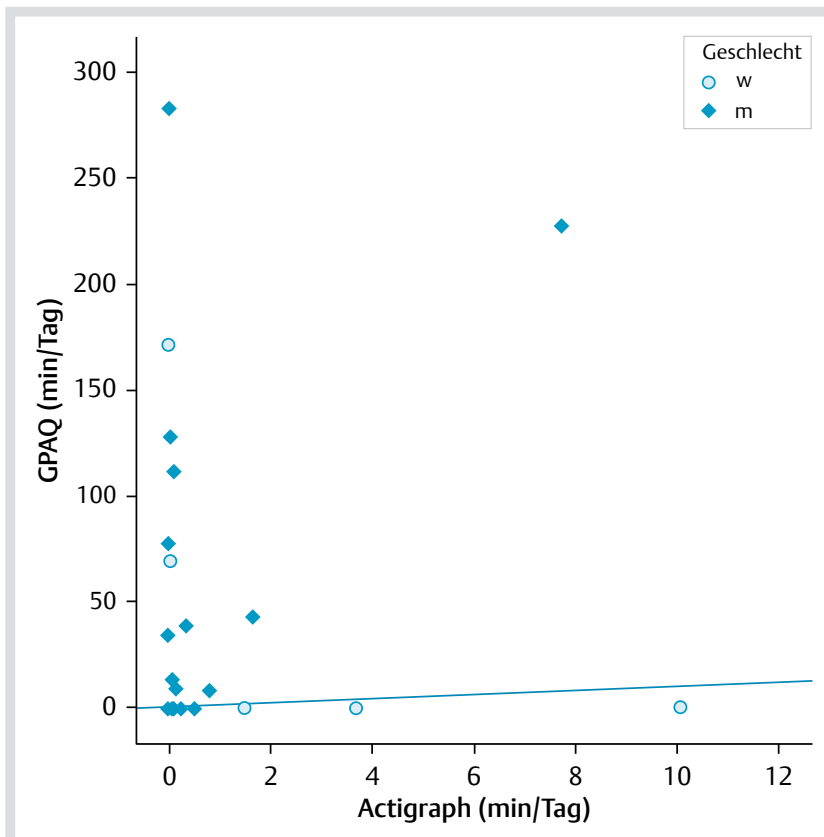

Abb. 5 Zusammenhang zwischen objektiver (Actigraph) und subjektiver (GPAQ) intensiver körperlicher Aktivität im Tagesmittel; Referenzintervall: $\pm 0,1 \mathrm{~min}(10 \%)$

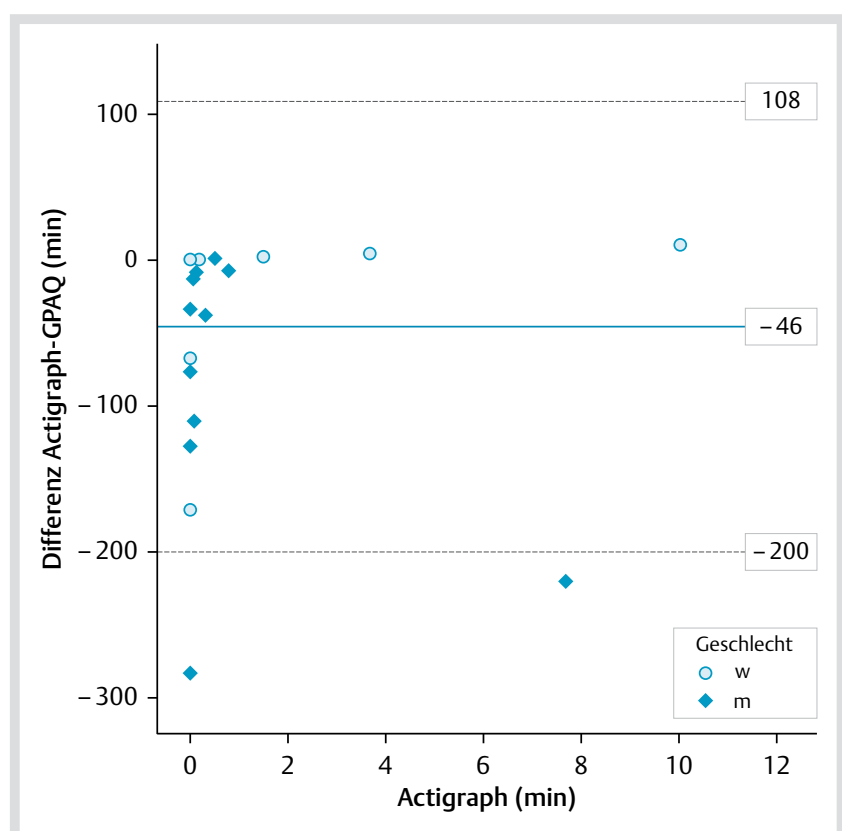

Abb. 6 Bland-Altman-Plot zur intensiven körperlichen Aktivität

nen (24\%) unterschätzten. Die Daten von 7 TeilnehmerInnen (28\%) lagen innerhalb des Intervalls. 10 Männer (58\%) bzw. 2 Frauen (25\%) überschätzten ihre intensive Aktivität.

- Abb. 6 stellt einen Bland-Altman Analyse zur Abweichung für die intensive körperliche Aktivität im Tagesmittel dar. Die mittlere Differenz zwischen den objektiven und subjektiven Angaben (Actigraph - GPAQ) betrug $-46 \mathrm{~min}(\mathrm{SD}=79)$, d. h. im Mittel überschätzen die TeilnehmerInnen die Dauer ihrer intensiven körperlichen Aktivität um 46 min pro Tag. Auffällig ist, dass die Streuung im Bereich niedriger objektiver Aktivität am größten ist. Es zeigen sich keine signifikanten Geschlechtsunterschiede in den Abweichungen $(\mathrm{p}=0,11)$.

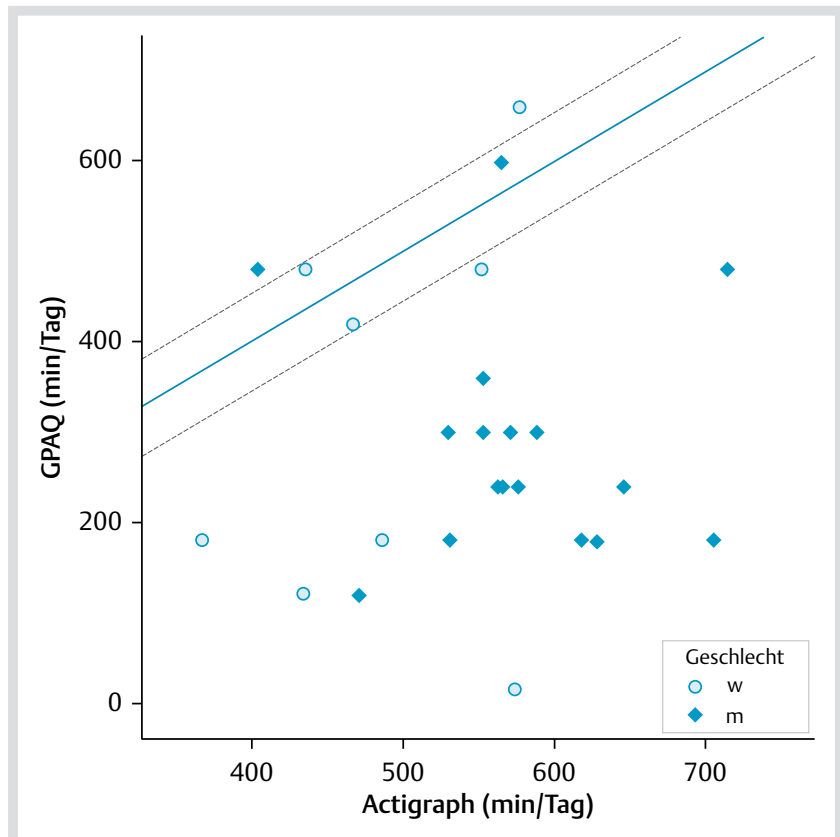

Abb. 7 Zusammenhang zwischen objektiver (Actigraph) und subjektiver (GPAQ) sitzender körperlicher (In-)Aktivität im Tagesmittel. Referenzintervall: $\pm 54,4 \min (10 \%)$

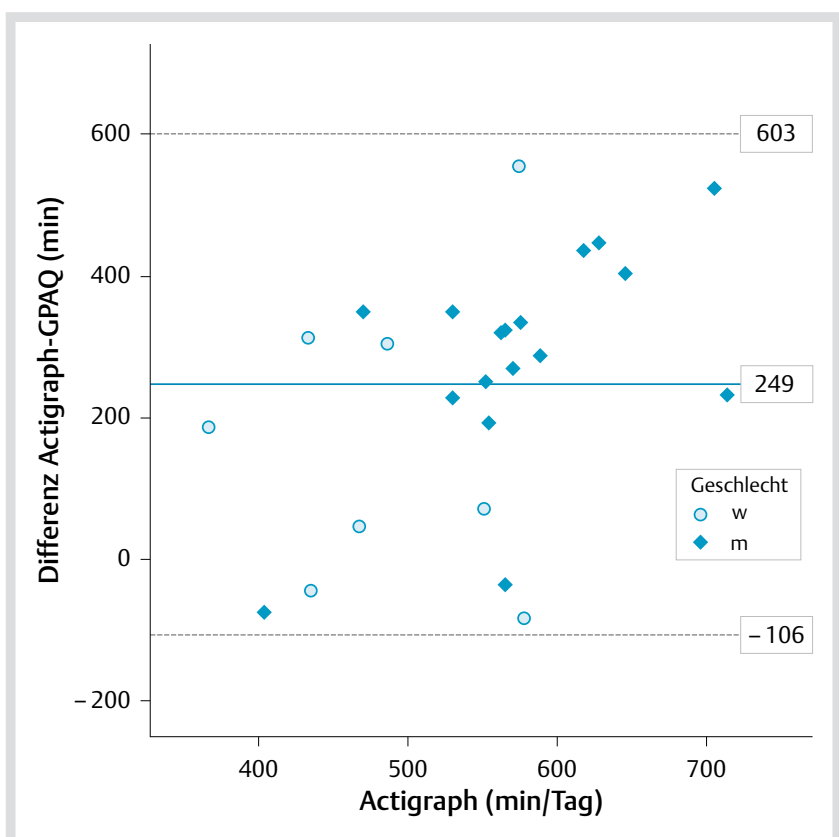

Abb. 8 Bland-Altman-Plot sitzender körperlicher (In-)Aktivität

Bezüglich der Sitzzeiten lag zwischen den subjektiven und objektiven Ergebnissen der Gesamtstichprobe eine sehr schwache Korrelation im Tagesmittel vor $(r=0,01)(\bullet$ Tab. 3).

- Abb. 7 stellt die Ergebnisse im Tagesmittel inklusive des Intervalls von $\pm 0 \%$ des objektiven Mittelwerts in einem Streudiagramm dar. Anhand des Intervalls zeigt sich, dass 3 TeilnehmerInnen (12\%) mit ihren Einschätzungen innerhalb des vorgegebenen Intervalls liegen. Insgesamt 20 TeilnehmerInnen (80\%) unterschätzen sich bezüglich ihrer täglichen Sitzzeiten, 2 überschätzen sich (8\%).

- Abb. 8 zeigt die Bland-Altman Analyse zu den Sitzzeiten. Die mittlere Differenz zwischen den objektiven und subjektiven An- 
gaben (Actigraph - GPAQ) betrug 249 min ( $S D=181$ ), d.h. im Mittel unterschätzen die TeilnehmerInnen die Dauer ihrer Sitzzeiten um mehr als 4 Stunden pro Tag. Die Abweichungen unterscheiden sich nicht signifikant zwischen männlichen und weiblichen Probanden $(p=0,11)$.

\section{Diskussion}

$\nabla$

Ziel dieser Studie war es, die Übereinstimmung von subjektiver und objektiver körperlicher Aktivität bei poststationären Rehabilitanden mit Rückenschmerzen zu vergleichen. Dabei zeigten sich keine signifikanten linearen Zusammenhänge zwischen subjektiver und objektiver Aktivität, sodass sich zusammenfassend sagen lässt, dass der GPAQ und der Actigraph nur geringe Übereinstimmungen zeigen. Die Bland-Altman Plots zeigen Überschätzungen der subjektiven Angaben im Vergleich zur objektiven Aktivität von $46 \mathrm{~min} /$ Tag (intensive Aktivität) bis $78 \mathrm{~min} /$ Tag (moderate Aktivität). Die Sitzzeiten werden hingegen um $249 \mathrm{~min} / \mathrm{Tag}$, d.h. mehr als 4 Stunden pro Tag, unterschätzt. Es zeigen sich keine geschlechtsspezifischen Unterschiede in den Abweichungen der Selbsteinschätzung.

Die vorliegenden Ergebnisse von Rückenpatienten entsprechen vergleichbaren Studien mit anderen Studienpopulationen und vergleichbaren Fragebogeninstrumenten. In der Literatur zeigen sich ebenfalls lediglich geringe bis moderate Übereinstimmungen mit Tendenz zur Überschätzung der objektiven körperlichen Aktivität [14,17,18,31-34]. Auch mehrere Reviews kamen zu dem Ergebnis einer mangelnden Übereinstimmung von subjektiver und objektiver körperlicher Aktivität $[13,15]$. Prince et al. [13] beschreiben dabei Tendenzen zu unterschiedlichen Abweichungen je nach Geschlecht und Aktivitätsbereich. Sie geben an, dass die Überschätzung im Bereich der intensiven Intensität am größten ist [13], was in den vorliegenden Ergebnissen ebenfalls zu erkennen ist. Die von Prince et al. [13] angemerkte Tendenz zu höheren Korrelationen zwischen subjektiver und objektiver Aktivität bei Männern findet sich in den vorliegenden Daten allerdings nicht. Im Gegensatz dazu fanden Hoos, Espinoza, Marshall und Arredondo [35] in ihrer Studie einen stärkeren Zusammenhang subjektiver und objektiver Aktivität im Bereich der intensiven Aktivitäten, wohingegen sie Schwierigkeiten bei der Einschätzung moderater Aktivitäten beschreiben [35]. Die Ergebnisse der vorliegenden Studie können dies allerdings nicht bestätigen. Da die objektiven Daten der vorliegenden Arbeit keine intensive Aktivität bei Männern und Frauen zeigen, gibt es Anlass zu hinterfragen, ob die Patienten aufgrund ihrer mangelnden Bewegungserfahrung überhaupt in der Lage sind, moderate und intensive Aktivität in der subjektiven Wahrnehmung zu unterscheiden. Es ist davon auszugehen, dass insbesondere bei körperlich inaktiven Personengruppen mit der Interpretation von subjektiven Angaben zur körperlichen Aktivität sehr zurückhaltend umgegangen werden muss. Altschuler et al. [36] weisen darauf hin, dass die Erhebung der Intensität körperlicher Aktivität durch Fragen nach der Schwere der Atmung bzw. Zunahme des Pulses, wie es auch beim GPAQ erfolgt, einen großen Interpretationsspielraum bietet. So könnten Personen mit niedrigem Fitnesslevel, die schneller außer Atem kommen, entsprechende Intensität eher überschätzen [36]. Aus dem gleichen Grund ist es auch möglich, dass körperlich aktive Personengruppen die Intensitäten ihrer Aktivitäten eher unterschätzen. Folglich gilt es bei der Interpretation von Selbstangaben zur körper- lichen Aktivität, insbesondere auch hinsichtlich der Bewertung von Intensitätsangaben, vorsichtig zu sein [21].

Im Vergleich mit weiteren indikationsbezogenen Studien, d.h. Studien mit Rückenschmerzpatienten, sind die vorliegenden Ergebnisse mit Ergebnissen von van Weering, Vollenbroek-Hutten und Hermens [19] vergleichbar. Auch in dieser Studie zeigt sich eine schwache Korrelation zwischen subjektiver und objektiver Aktivität bei Rückenpatienten. Auffällig ist jedoch, dass etwa $30 \%$ der Rückenpatienten ihr Aktivitätsverhalten unterschätzen, wohingegen es lediglich $14 \%$ überschätzen.

Huijnen et al. [37] untersuchten den Zusammenhang subjektiver und objektiver Aktivität bei chronischen Rückenpatienten unter Berücksichtigung von Schmerz und Depression. In der adjustierten Auswertung zeigte sich ein signifikanter moderater Zusammenhang, wobei die Diskrepanzen in der Selbsteinschätzung bei Patienten mit höheren Depressionswerten größer waren und eine Tendenz zur Unterschätzung zeigte [37].

Auffällig in den vorliegenden Daten sind die negativen Korrelationen bei Frauen, auch wenn diese nicht als signifikant bezeichnet werden können. Sowohl bei moderater als auch intensiver körperlicher Aktivität zeigen sich negative Assoziationen mittlerer Stärke (moderat: $-0,55$; intensiv: $-0,53$ ), was darauf hindeutet, dass Frauen dazu tendieren, ihre körperliche Aktivität mit zunehmender objektiver Aktivität geringer einzuschätzen. Während sich bei Männern bezogen auf die körperliche Aktivität zwar sehr schwache, jedoch positive Korrelationen zeigen (moderat: 0,06; intensiv: 0,02), stellt sich bezogen auf Sitzzeiten ein umgekehrtes Bild und bei Männern eine geringfügige negative Assoziation $(-0,22)$ dar. Damit schätzen Männer ihre Sitzzeit mit zunehmender objektiver Sitzzeit tendenziell geringer ein. Im Gegensatz dazu zeigt die positive Assoziation bei Frauen, dass eine längere Sitzzeit auch mit einer höheren subjektiven Einschätzung der Sitzzeit einher geht $(0,30)$. Auch wenn der Stichprobenumfang der vorliegenden Untersuchung sehr gering und nicht repräsentativ für die gewählte Indikationsgruppe ist, könnte die Frage nach geschlechtsspezifischen Unterschieden in der Selbsteinschätzung ein explorativer Ansatz für weitere Forschungsarbeiten in diesem Bereich sein.

Die Stärke der vorliegenden Studie liegt im parallelen Einsatz der subjektiven und objektiven Messinstrumente. Auch dass die Evaluation des Zusammenhangs objektiver und subjektiver Daten nicht nur mittels Korrelationen ausgewertet wurde, sondern das Ausmaß des Zusammenhangs ergänzend anhand der Bland-Altman Analyse dargestellt wurde, ist als eine Stärke der Studie anzusehen.

Eine Limitation stellt insbesondere die kleine Stichprobengröße dar. Auch die Erhebung objektiver Aktivität mittels Actigraph zeigt einige Limitationen. So können aufgrund der Positionierung des Akzelerometers an der Hüfte reine Aktivitäten der oberen Extremität und auch Radfahren nur unzureichend erfasst werden. Hinzu kommt, dass die Auswertung der Daten maßgeblich von den gewählten Auswertungsalgorithmen beeinflusst wird. In diesem Fall wurde für die Einteilung der Aktivitätsintensitäten der Algorithmus von Freedson [27] gewählt, da dieser in der Literatur am häufigsten verwendet wird.

Des Weiteren kann auch die Kategorisierung der TeilnehmerInnen anhand der Streudiagramme auf Grundlage des gewählten Toleranzintervalls als kritisch betrachtet werden, wobei insbesondere das aus praktischer Sicht sehr konservativ gewählte Toleranzintervall von $\pm 10 \%$ diskutiert werden kann. 


\section{Ausblick}

Die vorliegenden Ergebnisse unterstreichen die Herausforderungen bei der Erfassung körperlicher Aktivität. Je nach Fragestellung und Studienziel gilt es deshalb aus dem Kontinuum zwischen Präzision und Praktikabilität geeignete Messinstrumente auszuwählen. Bei subjektiven Instrumenten gilt es zudem, Aspekte der Über- bzw. Unterschätzung zu kontrollieren. Dafür erscheint zumindest in Subgruppen der jeweiligen Studienpopulation ein paralleler Einsatz von objektiven und subjektiven Verfahren empfehlenswert [14]. Insbesondere bei der Evaluation von Interventionsstudien mittels Fragebögen gilt es zu beachten, dass es aufgrund einer veränderten Selbsteinschätzung der Patienten zu Interventionsende zu Verzerrungen bei der Beurteilung von Interventionseffekten kommen kann. Folglich ist die Überprüfung des Zusammenhangs subjektiver und objektiver Aktivität zu unterschiedlichen Messzeitpunkten im Interventionsverlauf von großem Interesse.

Aus indikationsspezifischer Perspektive sind weitere Auswertungen von Interesse, um mögliche Zusammenhänge von Schmerzen bzw. Einschränkungen bei Alltagsaktivitäten und der aktivitätsbezogenen Selbsteinschätzung zu untersuchen.

Um konkrete Interventionsansätze für die therapeutische Praxis zur Verbesserung der aktivitätsbezogenen Selbsteinschätzung abzuleiten, ist zudem eine Längsschnittstudie zur Selbsteinschätzung körperlicher Aktivität vor und nach dem Rehabilitationsaufenthaltes von hoher Relevanz.

\section{Danksagung}

Wir danken der Deutschen Rentenversicherung Rheinland für die Förderung im Rahmen der Studie Bewegungscoaching. Wir danken der Aggertalklinik in Engelskirchen für die Kooperation im Rahmen der Bewegungscoaching Studie. Wir danken allen Patienten und Patientinnen für die Teilnahme.

\section{Interessenkonflikt}

Die Autoren geben keinen Interessenskonflikt an.

\section{Literatur}

1 Brüggemann S, Sewöster D. Bewegungstherapeutische Versorgung in der medizinischen Rehabilitation der Rentenversicherung. Bewegungstherapie und Gesundheitssport 2010; 26: 266-269

2 Pfeifer K, Sudeck G, Brüggemann $S$ et al. Bewegungstherapie in der medizinischen Rehabilitation - Wirkungen, Qualität, Perspektiven. Die Rehabilitation 2010; 49: 224-236

3 Bundesärztekammer, Kassenärztliche Bundesvereinigung, Arbeitsgemeinschaft der Wissenschaftlichen Medizinischen Fachgesellschaften. Nationale Versorgungsleitlinie Kreuzschmerz. [Online] 2013; [Zitat vom 30. November 2014] http://www.leitlinien.de/nvl/kreuzschmerz

4 Abenhaim L, Rossignol M, Valat JP et al. The role of activity in the therapeutic management of back pain. Report of the International Paris Task Force on Back Pain. Spine 2000; 25: 1-33

5 van Tulder M, Becker A, Bekkering T et al. Chapter 3. European guidelines for the management of acute nonspecific low back pain in primary care. Eur Spine J 2006; 15: 169-191

6 Ronda G, van Assema P, Brug J. Stages of change, psychological factors and awareness of physical activity levels in The Netherlands. Health promotion international 2001; 16: 305-314

7 Lechner L, Bolman C, van Dijke M. Factors related to misperception of physical activity in The Netherlands and implications for health promotion programmes. Health promotion international 2006; 21: 104-112
8 Watkinson C, van Sluijs EMF, Sutton S et al. Overestimation of physical activity level is associated with lower BMI: a cross-sectional analysis. The international journal of behavioral nutrition and physical activity 2010; 7: 68

9 van Sluijs EMF, Griffin SJ, van Poppel MNM. A cross-sectional study of awareness of physical activity: associations with personal, behavioral and psychosocial factors. The international journal of behavioral nutrition and physical activity 2007; 4: 53

10 Müller C, Winter C, Rosenbaum D. Aktuelle objektive Messverfahren zur Erfassung körperlicher Aktivität im Vergleich zu subjektiven Erhebungsmethoden. DZSM 2010; 61: 11-18

11 Corder $K$, Ekelund $U$, Steele RM et al. Assessment of physical activity in youth. J Appl Physiol 2008; 105: 977-987

12 Beneke R, Leithäuser RM. Körperliche Aktivität im Kindesalter - Messverfahren. DZSM 2008; 59: 215-222

13 Prince SA, Adamo KB, Hamel ME et al. A comparison of direct versus self-report measures for assessing physical activity in adults: a systematic review. The international journal of behavioral nutrition and physical activity 2008; 5: 56

14 Gaede-Illig C, Alfermann D, Zachariae S et al. Körperliche Aktivität erfassen - ein Vergleich vom IPAQ-SF und dem SenseWear Pro Armband. DZSM 2014; 65: 154-159

15 Lee PH, Macfarlane DJ, Lam TH et al. Validity of the International Physical Activity Questionnaire Short Form (IPAQ-SF): a systematic review. The international journal of behavioral nutrition and physical activity 2011; 8: 115

16 Celis-Morales CA, Perez-Bravo F, Ibañez L et al. Objective vs. self-reported physical activity and sedentary time: effects of measurement method on relationships with risk biomarkers. PloS one 2012; 7: e36345

17 Grimm EK, Swartz AM, Hart T et al. Comparison of the IPAQ-Short Form and accelerometry predictions of physical activity in older adults. Journal of aging and physical activity 2012; 20: 64-79

18 Lagersted-Olsen J, Korshøj M, Skotte J et al. Comparison of objectively measured and self-reported time spent sitting. Int J Sports Med 2014; 35: $534-540$

19 van Weering MGH, Vollenbroek-Hutten MMR, Hermens HJ. The relationship between objectively and subjectively measured activity levels in people with chronic low back pain. Clin Rehabil 2011; 25: 256-263

20 Schaller A, Froboese I. Movement coaching: study protocol of a randomized controlled trial evaluating effects on physical activity and participation in low back pain patients. BMC musculoskeletal disorders 2014; 15: 391

21 Troiano RP, Berrigan D, Dodd KW et al. Physical activity in the United States measured by accelerometer. Med Sci Sports Exerc 2008; 40: 181-188

22 Hagströmer $M$, Troiano RP, Sjöström $M$ et al. Levels and patterns of objectively assessed physical activity - a comparison between Sweden and the United States. Am J Epidemiol 2010; 171: 1055-1064

23 Department für Prävention von chronischen Krankheiten. Global Physical Activity Questionnaire (GPAQ). Der „STEPwise ap-proach“ zur Surveillance von Risikofaktoren für chronische Krankheiten. Genf: WHO; 2006

24 Armstrong T, Bull F. Development of the World Health Organization Global Physical Activity Questionnaire (GPAQ). J Public Health 2006; 14: $66-70$

25 Bull FC, Maslin TS, Armstrong T. Global physical activity questionnaire (GPAQ): nine country reliability and validity study. Journal of physical activity \& health 2009; 6: 790-804

26 Cleland $C L$, Hunter RF, Kee $F$ et al. Validity of the global physical activity questionnaire (GPAQ) in assessing levels and change in moderatevigorous physical activity and sedentary behaviour. BMC public health 2014; 14: 1255

27 Freedson PS, Melanson E, Sirard J. Calibration of the Computer Science and Applications, Inc. accelerometer. Med Sci Sports Exerc 1998; 30: 777-781

28 Troiano RP. Large-scale applications of accelerometers: new frontiers and new questions. Med Sci Sports Exerc 2007; 39: 1501

29 Bland JM, Altman DG. Statistical methods for assessing agreement between two methods of clinical measurement. Lancet 1986; $1: 307-310$

30 Krouwer JS. Why Bland-Altman plots should use $\mathrm{X}$, not $(\mathrm{Y}+\mathrm{X}) / 2$ when $\mathrm{X}$ is a reference method. Stat Med 2008; 27: 778-780

31 de Cocker K, Cardon G, de Bourdeaudhuij I. Pedometer-determined physical activity and its comparison with the International Physical Activity Questionnaire in a sample of Belgian adults. Res Q Exerc Sport 2007; 78: 429-437

32 Craig CL, Marshall AL, Sjöström $M$ et al. International physical activity questionnaire: 12-country reliability and validity. Med Sci Sports Exerc 2003; 35: 1381-1395 
33 Ekelund U, Sepp $H$, Brage $S$ et al. Criterion-related validity of the last 7-day, short form of the International Physical Activity Questionnaire in Swedish adults. Public Health Nutrition 2006; 9: 258-265

34 Rzewnicki R, Vanden Auweele Y, de Bourdeaudhuij I. Addressing overreporting on the International Physical Activity Questionnaire (IPAQ) telephone survey with a population sample. Public Health Nutrition 2003; 6: 299-305

35 Hoos T, Espinoza N, Marshall S et al. Validity of the Global Physical Activity Questionnaire (GPAQ) in adult Latinas. Journal of physical activity \& health 2012; 9: 698-705
36 Altschuler A, Picchi T, Nelson M et al. Physical activity questionnaire comprehension: lessons from cognitive interviews. Med Sci Sports Exerc 2009; 41: 336-343

37 Huijnen IPJ, Verbunt JA, Peters ML et al. Do depression and pain intensity interfere with physical activity in daily life in patients with Chronic Low Back Pain? Pain 2010; 150: 161-166 